\title{
Computational Study of Peptide Plane Stacking with Polar and Ionizable Amino Acid Side Chains
}

\author{
Yefei Wang, Jia Wang, and Lishan Yao* \\ Laboratory of Biofuels, Qingdao Institute of Bioenergy and Bioprocess Technology, Chinese Academy of Sciences, No. 189 Songling \\ Road, Laoshan District, Qingdao 266061, China
}

\section{Supporting Information}

ABSTRACT: Parallel and T-shaped stacking interactions of the peptide plane with polar and ionizable amino acid side chains (including aspartic/ glutamic acid, asparagine/glutamine, and arginine) are investigated using the quantum mechanical MP2 and CCSD computational methods. It is found that the electrostatic interaction plays an essential role in determining the optimal stacking configurations for all investigated stacking models. For certain complexes, the dispersion interaction also contributes considerably to stacking. In the gas phase, the stacking interaction of the charged system is stronger than that of the neutral

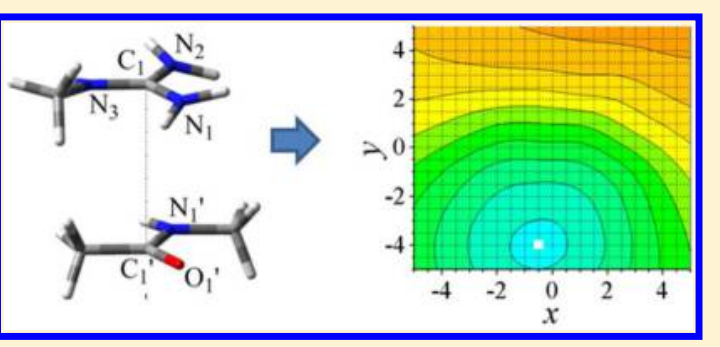
system, and T-shaped stacking is generally more preferred than parallel stacking, with the stacking energy in the range of -4 to $-18 \mathrm{kcal} / \mathrm{mol}$. The solvation effect overall weakens stacking, especially for the charged system and the T-shaped stacking configurations. In water, the interaction energies of different stacking models are comparable.

\section{INTRODUCTION}

$\pi$ interactions are an important type of noncovalent interactions in molecules and essential in chemistry and biology. ${ }^{1-6} \pi$ interactions, involving $\pi$ electrons, can be divided into different subtypes, for example, $\pi-\pi{ }^{1}$ anion $-\pi,^{2}$ cation $-\pi,^{7-9}$ and $\mathrm{XH}-\pi^{10-12}$ depending on the components of the system. Accurate binding energies can be retrieved from high-level quantum mechanical calculations, for example, the $\operatorname{CCSD}(\mathrm{T}) /$ complete basis set (CBS) limit, ${ }^{4,13}$ which allows a direct comparison of different types of $\pi$ interactions. The binding energy can be decomposed to four terms, electrostatic, exchange repulsion, polarization, and dispersion. ${ }^{14-16}$ The electrostatic term describes the interaction of electric multipoles; the polarization term is the interaction between the permanent multipoles and the induced multipoles; the dispersion term describes the interaction between the instantaneous multipoles and the induced multipoles. Taking benzene dimer as an example, the dispersion and electrostatic terms contribute the most to the interaction energy. ${ }^{17-20}$ Compared with the $\sigma$-electron system, the $\pi$-electron system has a high polarizability due to the delocalization property of the $\pi$ electrons. ${ }^{21}$ Thus, the polarization energy can be significant in the cation $-\pi$ and anion $-\pi$ systems, ${ }^{2,22,23}$ whereas the dispersion energy is important when the complex is neutral.

For proteins, interactions with aromatic amino acid side chains, including phenylalanine, tyrosine, and tryptophan, can be very important for their stability and function. ${ }^{24-30}$ Molecular dynamics (MD) simulations showed that the miniprotein Trp-cage ${ }^{31}$ with a tryptophan (W5) in the hydrophobic core cannot fold properly if the dispersion term is turned off, ${ }^{28}$ and the protein refolds if the dispersion term is restored. The stabilization from the aromatic residues to the protein structure comes from two aspects, the entropic (hydrophobic) effect and the enthalpic effect (direct interactions). Quantum mechanical (QM) calculations suggest that the enthalpic effect plays a more important role for the protein rubredoxin stability. ${ }^{24,25}$

Most studies of $\pi$ interactions in proteins focus on the aromatic side chains. Recently, stacking with the protein backbone amide has been investigated as well. ${ }^{32,33}$ The intramolecular peptide plane stacking is competitive with the hydrogen bonding. ${ }^{33,34}$ Compared with a benzene ring, the backbone amide plane has a large dipole moment, underlining the importance of electrostatics in the amide stacking interactions. QM calculations showed that the interaction energy between $\mathrm{N}$-methylacetamide (NMA) and the aromatic ring can be as large as $-5 \mathrm{kcal} / \mathrm{mol}$, depending on the dipole moment of the aromatic ring. ${ }^{32}$ This binding energy is more negative than that of the benzene dimer, $-2.7 \mathrm{kcal} / \mathrm{mol} .{ }^{35}$ Our previous study demonstrated that stacking of the E305 carboxylate, a buried side chain, with two peptide plane amides is important for the stability of Trichoderma reesei Cel5A. ${ }^{36} \mathrm{~A}$ survey of the protein structures in the pdb databank suggests that the parallel peptide backbone amide stacking with aspartate/glutamate has an occurrence frequency of 28 per 100 structures. $^{36}$ Ionizable residues inside proteins play an important role in protein functions, such as ligand binding, protein stability, and enzyme catalysis. It has been shown that electrostatic interactions are critical for the stabilization of the ionizable residues; ${ }^{37-42}$ however, stacking with the peptide

Received: January 8, 2015

Revised: $\quad$ March 18, 2015

Published: March 31, 2015 
plane, which can be important for the ionizable side-chain stability as well, has not been thoroughly investigated.

In this study, we focus on peptide amide stacking with aspartate/glutamate (both protonated (DE) and deprotonated $\left.\left(\mathrm{DE}^{-}\right)\right)$, asparagine/glutamine (NQ), and arginine side chains $\left(\mathrm{R}^{+}\right)$using $\mathrm{QM}$ calculations. We characterize the peptide plane stacking preferences with different groups, the role of the charge distribution, and the solvation effect. The optimal stacking configurations are identified through scanning the interaction energy at the $\mathrm{MP} 2 / 6-31+\mathrm{G}(\mathrm{d}, \mathrm{p})$ level. ${ }^{43}$ Then, these interaction energies are decomposed by the localized molecular orbital energy decomposition analysis (LMOEDA), ${ }^{14}$ a very efficient method that provides more insights into the property of stacking. The results show that the electrostatic interaction plays a dominant role in the stacking orientation and position especially for the charged system. For the neutral system, the dispersion interaction contributes considerably to stacking. For some T-shaped complexes, the hydrogen bond can be formed. In the gas phase, stacking of the charged system is much stronger than that of the neutral system. The solvation effect generally weakens stacking for both systems, with the charged system interaction affected more significantly. As a result, the interaction energies are similar for the charged and the neutral systems in water.

\section{METHODS AND MATERIALS}

The truncated molecular fragments were used to model the $\pi-\pi$ interactions between the peptide amide plane and the amino acid side chains. Specifically, the peptide plane was modeled using NMA, while aspartate/glutamate $\left(\mathrm{DE}^{-}\right)$, aspartic/glutamic acid (DE), asparagine/glutamine (NQ),or arginine $\left(\mathrm{R}^{+}\right)$was truncated to a functional group saturated with a methyl (Figure 1). With the geometries of the stacking

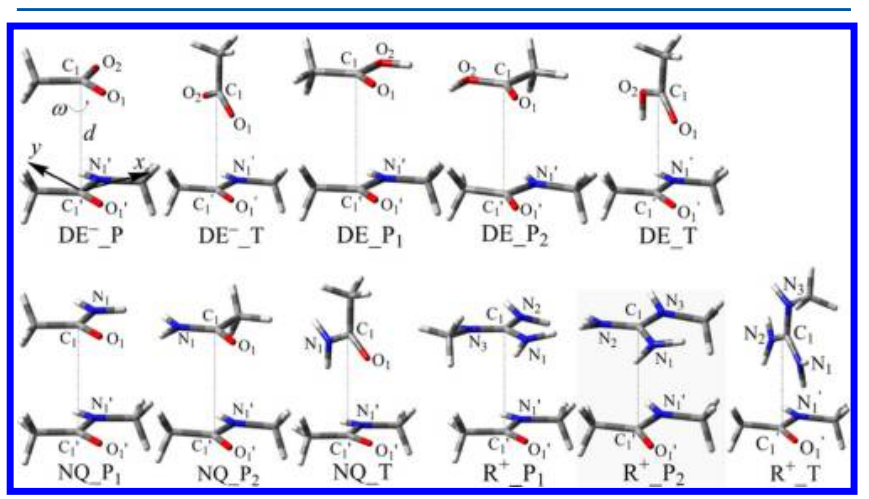

Figure 1. Diagram of $\mathrm{DE}^{-}-, \mathrm{DE}-, \mathrm{NQ}-$, and $\mathrm{R}^{+}-\mathrm{NMA}$ dimers. The $x y$ plane is defined by the three atoms, $\mathrm{N}_{1}{ }^{\prime}, \mathrm{C}_{1}{ }^{\prime}$, and $\mathrm{O}_{1}{ }^{\prime}$ of NMA, with the $y$ axis along the bond vector $\mathrm{O}_{1}{ }^{\prime}-\mathrm{C}_{1}{ }^{\prime}$. $d$ is defined as the distance between the atom $\mathrm{C}_{1}$ and its projection on the $x y$. The dihedral $\omega$ is defined as $\angle \mathrm{O}_{1}-\mathrm{C}_{1}-\mathrm{C}_{1}{ }^{\prime}-\mathrm{O}_{1}{ }^{\prime}$ for $\mathrm{DE}^{-}, \mathrm{DE}$, and $\mathrm{NQ}$ or $\angle \mathrm{N}_{1}-\mathrm{C}_{1}-$ $\mathrm{C}_{1}{ }^{\prime}-\mathrm{O}_{1}{ }^{\prime}$ for $\mathrm{R}^{+}$.

components optimized at the MP2/6-31+G(d,p) level, ${ }^{43,44}$ the parallel and T-shaped NMA-amino acid side-chain models were constructed by arranging the two planes of the monomers parallelly or perpendicularly. The plane was defined by three atoms, that is, $\mathrm{O}_{1}{ }^{\prime}-\mathrm{C}_{1}{ }^{\prime}-\mathrm{N}_{1}{ }^{\prime}$ for NMA, $\mathrm{O}_{1}-\mathrm{C}_{1}-\mathrm{O}_{2}$ for $\mathrm{DE}$ and $\mathrm{DE}^{-}, \mathrm{O}_{1}-\mathrm{C}_{1}-\mathrm{N}_{1}$ for $\mathrm{NQ}$ and $\mathrm{N}_{1}-\mathrm{C}_{1}-\mathrm{N}_{2}$ for $\mathrm{R}^{+}$(Figure 1). For parallel stacking with $\mathrm{DE}, \mathrm{NQ}$ and $\mathrm{R}^{+}$, two mirror structures resulted in two different configurations, which were denoted as $\mathrm{P}_{1}$ and $\mathrm{P}_{2}$. A 3-D potential energy surface (PES) was constructed at the MP2/6-31+G(d,p) level for each complex as follows. By defining $\mathrm{N}_{1}{ }^{\prime}-\mathrm{C}_{1}{ }^{\prime}-\mathrm{O}_{1}{ }^{\prime}$ of $\mathrm{NMA}$ as the $x y$ plane (with $\mathrm{C}_{1}{ }^{\prime}$ as origin) and restricting the distance between $\mathrm{C}_{1}$ and the $x y$ plane $(d)$ to $4.0 \AA$, the dihedral $\omega$ (defined as $\angle \mathrm{O}_{1}-\mathrm{C}_{1}-$ $\mathrm{C}_{1}{ }^{\prime}-\mathrm{O}_{1}{ }^{\prime}$ for $\mathrm{DE}^{-}, \mathrm{DE}$, and $\mathrm{NQ}$ or $\angle \mathrm{N}_{1}-\mathrm{C}_{1}-\mathrm{C}_{1}{ }^{\prime}-\mathrm{O}_{1}{ }^{\prime}$ for $\mathrm{R}^{+}$) was rotated around the $Z$ axis by $30.0^{\circ}$ increments (Figure 1 ). Simultaneously, the projection of $\mathrm{C}_{1}$ on the $x y$ plane (with the coordinate $(x, y))$ was scanned by sampling grid points every $0.5 \AA$ (from -5 to $+5 \AA$ ) where the $+y$ direction was along the $\mathrm{O}_{1}{ }^{\prime}=\mathrm{C}_{1}{ }^{\prime}$ bond vector. After the optimal configuration was obtained, the $d$ distance was scanned in $0.1 \AA$ increments. The starting configuration of each complex and the geometric variables are shown in Figure 1. All of the interaction energies $(\Delta E)$ were counterpoise-corrected for the basis set superposition error (BSSE). ${ }^{45}$

The binding energies of the optimal configurations were also calculated by MP2/CBS ${ }^{43,44}$ (complete basis set) and CCSD(T)/CBS to validate the obtained PESs. The MP2/CBS energies were determined with the use of aug-cc-pVnZ $(n=\mathrm{D}$, $\mathrm{T})^{46,47}$ basis sets and the Helgaker's extrapolation scheme. ${ }^{48}$ The $\operatorname{CCSD}(\mathrm{T}) / \mathrm{CBS}$ energies were obtained by adding the difference between the $\mathrm{MP} 2 / 6-31+\mathrm{G}(\mathrm{d}, \mathrm{p})$ and $\operatorname{CCSD}(\mathrm{T}) / 6$ $31+\mathrm{G}(\mathrm{d}, \mathrm{p})$ energies to the $\mathrm{MP} 2 / \mathrm{CBS}$ values. ${ }^{30}$ The total interaction energies were decomposed to electrostatic, exchange-repulsion, polarization, and dispersion components by the LMO-EDA analysis to reveal the contributions of various intermolecular interactions. ${ }^{14}$ The polarizable continuum model (PCM $)^{49}$ with different dielectric constants (4.0 and 80.0 ) was adopted to investigate the solvation effect on binding energies. All calculations were performed using the Gaussian $09^{50}$ and Gamess ${ }^{43,51-53}$ software packages.

A total of 6338 high-quality protein structures were selected from the pdb databank (resolution $<2.0 \AA$, $R<0.3$, chain length $>100$ ) using the program PISCES. ${ }^{54}$ The sequence identity between pair structures was limited to $<25 \%$. The amide (of glutamine and asparagine) peptide plane stacking as well as the guanidino group (of arginine) peptide plane stacking from the selected structures were identified using an in-housing $\mathrm{VMD}^{55}$ script.

\section{RESULTS AND DISCUSSION}

PES Scan. The 3-D $(x, y, \omega) \Delta E$ binding PES at the MP2/6$31+\mathrm{G}(\mathrm{d}, \mathrm{p})$ level was constructed for $\mathrm{DE}^{-}-, \mathrm{DE}-, \mathrm{NQ}-$, and $\mathrm{R}^{+}-\mathrm{NMA}$ with the fragment distance $d$ fixed at $4.0 \AA$. (The geometric parameters are defined in Figure 1.) After the $(x, y$, $\omega)$ values of the energy minimum state were identified, the distance $d$ was then scanned with $(x, y, \omega)$ fixed to determine the optimal stacking configuration (Table 1). The $\operatorname{CCSD}(\mathrm{T}) /$ CBS binding energy values were also calculated for the optimal configurations of different complexes and correlated well with the MP2/6-31+G(d,p) data (Figure 2A). The CCSD $(T) / C B S$ calculations validate the PES scan at the MP2/6-31+G(d,p) level. The LMO-EDA energy decomposition analysis was also performed at MP2/aug-cc-pVnZ $(n=\mathrm{D}, \mathrm{T})$ levels for optimal configurations (Figure 2B). As can be seen, different energy terms at $\mathrm{MP} 2 / 6-31+\mathrm{G}(\mathrm{d}, \mathrm{p})$ agree quite well with the MP2/ aug-cc-pVnZ $(n=\mathrm{D}, \mathrm{T})$ results and those extrapolated at MP2/ CBS and $\operatorname{CCSD}(\mathrm{T}) / \mathrm{CBS}$, except for the fact that the dispersion term is underestimated by $\sim 1 \mathrm{kcal} / \mathrm{mol}$, which explains the intercept of $-1.2 \mathrm{kcal} / \mathrm{mol}$ in Figure $2 \mathrm{~A}$.

Optimal Configurations of the Parallel Stacking. Figure 3 and Figure S1 in the Supporting Information (SI) shows the 2-D $(x, y)$ PES contour plot with the $\omega$ dihedral 
Table 1. Components of the MP2/6-31+G(d,p) Interaction Energy for Optimal Configurations in the Gas Phase (kcal/mol)

\begin{tabular}{|c|c|c|c|c|c|c|}
\hline & $(x, y, \omega, d)(\AA, \AA, \operatorname{deg}, \AA)$ & $\Delta E_{\text {elec }}$ & $\Delta E_{\text {exre }}$ & $\Delta E_{\text {pola }}$ & $\Delta E_{\text {disp }}$ & $\Delta E_{\text {total }}$ \\
\hline DE_P $P_{1}$ & $(1.0,0.0,120,3.4)$ & -3.7 & 3.6 & -0.7 & -2.3 & -3.1 \\
\hline DE_P $P_{2}$ & $(0.5,0.0,210,3.5)$ & -3.4 & 2.6 & -0.6 & -1.7 & -3.1 \\
\hline NQ $P_{1}$ & $(-0.5,0.5,180,3.6)$ & -3.7 & 2.8 & -0.6 & -2.0 & -3.6 \\
\hline $\mathrm{NQ} \mathrm{P}_{2}$ & $(1.5,0.0,150,3.6)$ & -3.2 & 2.7 & -0.6 & -2.2 & -3.3 \\
\hline $\mathrm{DE}^{-} \mathrm{P}$ & $(1.5,2.0,120,3.4)$ & -6.5 & 3.9 & -1.8 & -1.9 & -6.3 \\
\hline $\mathrm{R}^{+} \mathrm{P}_{1}$ & $(-0.5,-4.0,240,2.8)$ & -10.9 & 5.9 & -2.9 & -1.7 & -9.6 \\
\hline $\mathrm{R}_{-}^{+} \mathrm{P}_{2}$ & $(-0.5,-4.0,90,3.5)$ & -7.8 & 3.5 & -1.9 & -1.2 & -7.5 \\
\hline DE_T & $(0.0,-0.5,180,3.6)$ & -10.5 & 8.1 & -2.7 & -1.0 & -6.1 \\
\hline NQT & $(0.0,-0.5,180,3.7)$ & -9.5 & 6.9 & -2.5 & -1.1 & -6.2 \\
\hline $\mathrm{DE}_{-}^{-} \mathrm{T}$ & $(1.0,3.0,90,2.2)$ & -21.2 & 15.5 & -8.0 & -2.0 & -15.6 \\
\hline $\mathrm{R}_{-}^{+} \mathrm{T}$ & $(-0.5,-2.5,120,3.4)$ & -19.1 & 11.9 & -7.6 & -1.2 & -15.9 \\
\hline
\end{tabular}

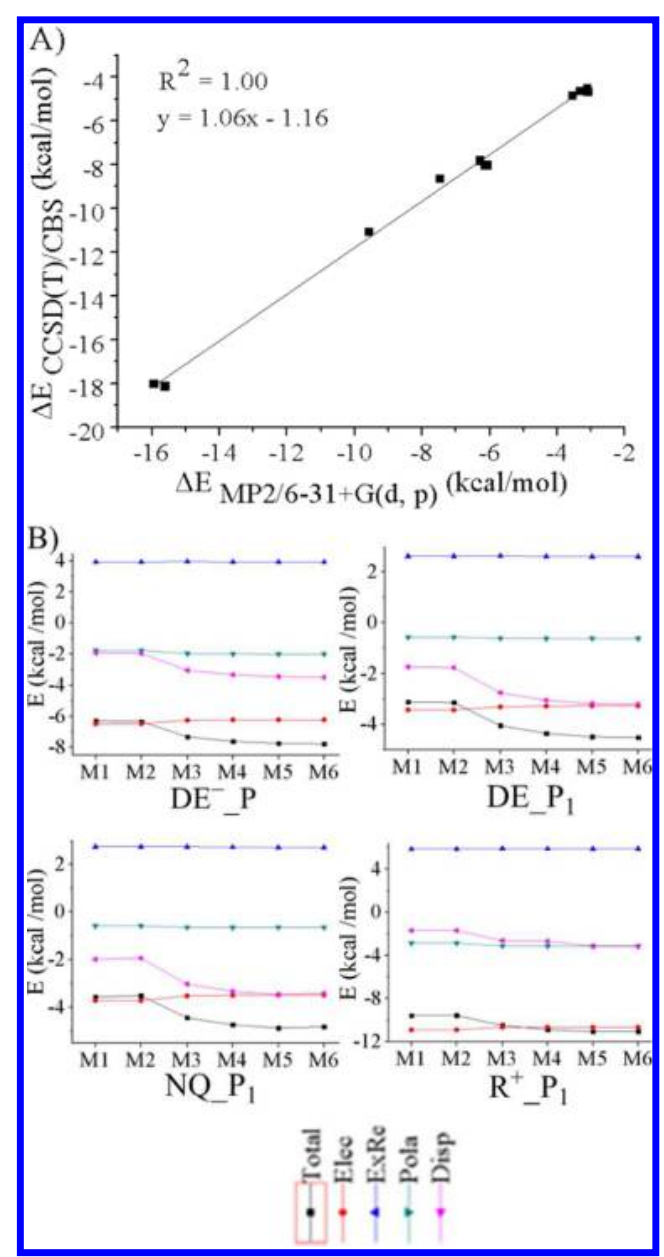

Figure 2. (A) Correlation between the MP2/6-31+G(d,p) binding energy and the $\operatorname{CCSD}(\mathrm{T}) / \mathrm{CBS}$ binding energy for the optimal configurations. (B) LMO-EDA energy decomposition using different methods: MP2 $/ 6-31+\mathrm{G}(\mathrm{d}, \mathrm{p})(\mathrm{M} 1), \mathrm{CCSD}(\mathrm{T}) / 6-31+\mathrm{G}(\mathrm{d}, \mathrm{p})(\mathrm{M} 2)$, MP2/aug-cc-pVDZ (M3), MP2/aug-cc-pVTZ (M4), MP2/CBS (M5), and $\operatorname{CCSD}(\mathrm{T}) / \mathrm{CBS}(\mathrm{M} 6)$.

angle value the same as that of the optimal configuration in the searched space and $d=4.0 \AA$. The optimal configuration for each parallel stacking model is shown in Figure 4. Considering that all stacking components are charged or polar, we attempt to rationalize the optimal stacking configurations in terms of electrostatics. The electrostatic potentials (ESPs) and dipole moment vectors of all components are shown in Figure 5. Taking the $\mathrm{DE}^{-}$as an example, the ESPs around all atoms are negative, and the most negative areas are centered on $\mathrm{O}_{1}$ and

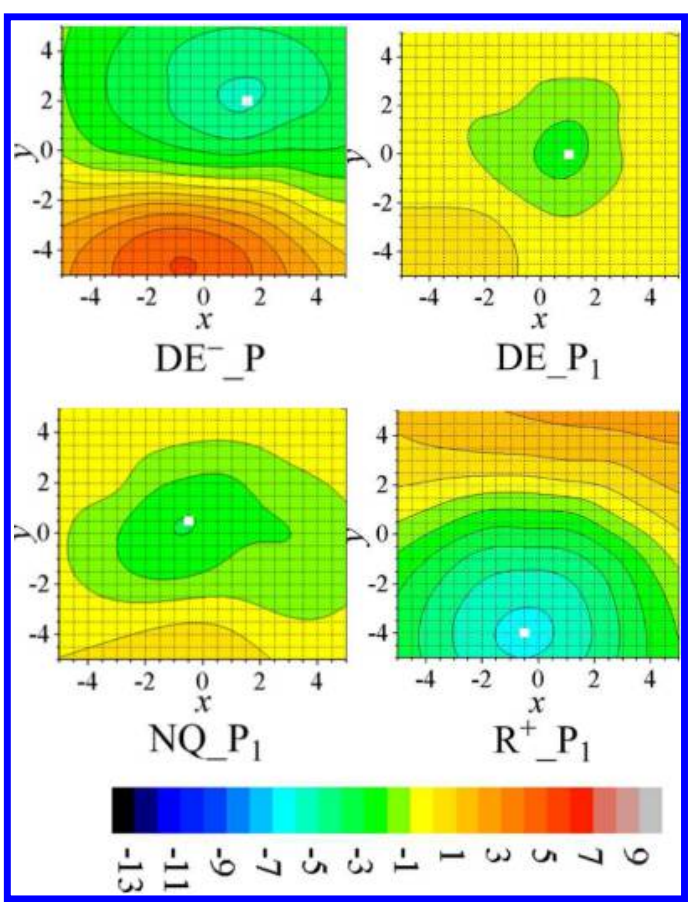

Figure 3. MP2 $/ 6-31+\mathrm{G}(\mathrm{d}, \mathrm{p})(x, y)$ PES that contains the binding energy minimum of the parallel dimer, with each contour corresponding to $1 \mathrm{kcal} / \mathrm{mol}$. The minimum energy site was marked with a white square.

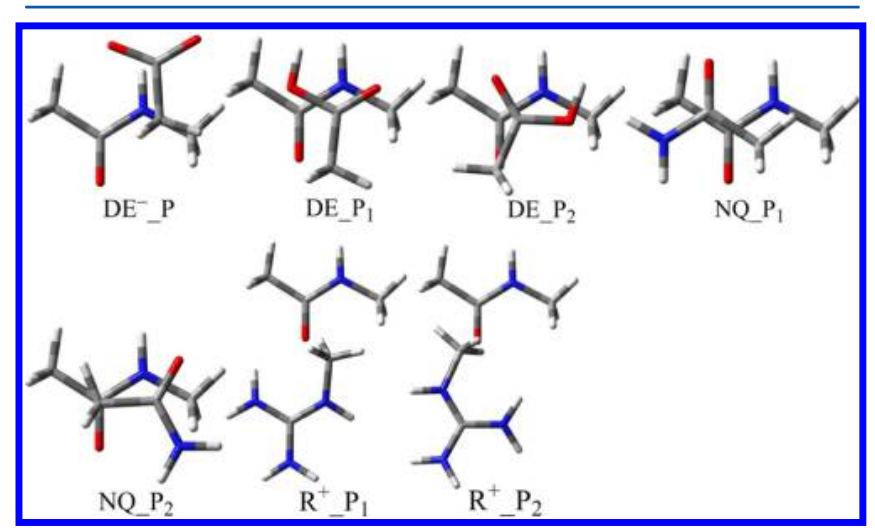

Figure 4. Optimal parallel complexes obtained at the MP2/6$31+\mathrm{G}(\mathrm{d}, \mathrm{p})$ level $(d=4.0 \AA)$.

$\mathrm{O}_{2}$. The $\mathrm{DE}^{-}$carboxyl group tends to locate above the most positive ESP area of NMA (around $\mathrm{N}_{1}{ }^{\prime} \mathrm{H}$, Figures 1, 4, and 5) with the corresponding $(x, y)$ coordinates of $(1.5 \AA, 2.0 \AA)$ 


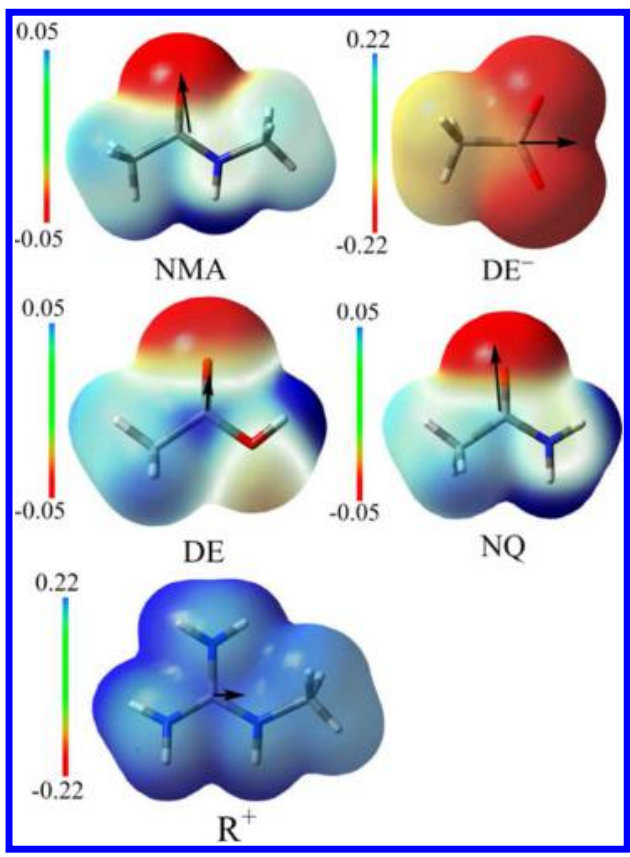

Figure 5. Molecular electrostatic potentials (EPSs) and the dipole moment vectors for all monomers optimized at MP2/6-31+G(d,p).

(Table 1). As shown in Figure 5, the dipole of NMA is approximately along the $\mathrm{C}_{1}{ }^{\prime}-\mathrm{O}_{1}{ }^{\prime}$ vector (tilted by $12^{\circ}$ ), while the dipole of $\mathrm{DE}^{-}$points along the bisector of the angle $\angle \mathrm{O}_{1}-$ $\mathrm{C}_{1}-\mathrm{O}_{2}$. In the optimal $\mathrm{DE}^{-}-\mathrm{NMA}$ parallel stacking ( $\mathrm{DE}^{-} \mathrm{P}$, $\left.\omega=120^{\circ}\right)($ Table 1$)$, the two dipoles are aligned antiparallelly, suggesting that the orientation is determined by the dipoledipole interaction (Figure 4). To confirm this, we extracted the interaction energy at each $\omega$ (with $(x, y)$ fixed at (1.5., $2.0 \AA)$ ) from the 3-D PES (Figure 6 and Figure S2 in the SI). As can be seen, the rotation of the $\mathrm{DE}^{-}$dipole leads to $\sim 1.7 \mathrm{kcal} / \mathrm{mol}$ variation of the interaction energy. The LMO-EDA analysis

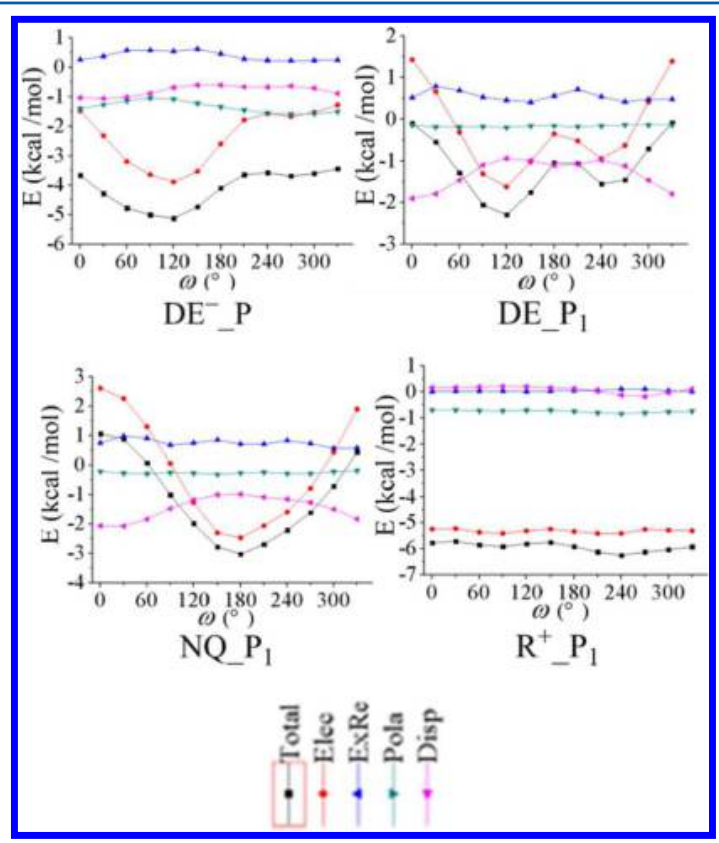

Figure 6. Energy components as a function of $\omega$ with $(x, y)$ coordinates fixed at the optimal values and $d=4.0 \AA \AA$ (Table 1). The curves were obtained from the MP2/6-31+G(d,p) calculations. shows that the electrostatic energy profile is similar to that of the total binding energy, whereas the other three terms have much smaller variations (Figure 6 and Figure S2 in the SI). As reported in Table 1, the electrostatic term dominates the total interaction energy for the $\mathrm{DE}^{-} \mathrm{P}$ optimal complex configuration $\left((x, y, \omega, d)\right.$ of $\left.\left(1.5 \AA, 2.0 \AA, 120^{\circ}, 3.4 \AA\right)\right)$.

For the DE-NMA complex DE_ $\mathrm{P}_{1}$ and $\mathrm{DE}_{-} \mathrm{P}_{2}$ (Figure 1), the absolute values of the binding energy are considerably smaller than that of $\mathrm{DE}^{-}-\mathrm{NMA}$ (Figure 3 ). The ESPs of DE have both negative and positive values (Figure 5). Specifically, the areas around $\mathrm{O}_{1}$ and $\mathrm{O}_{2}$ atoms are negative, while the other areas are positive. The LMO-EDA analysis of the optimal DENMA configuration $\left(\left(1.0 \AA, 0.0 \AA, 120^{\circ}, 3.4 \AA\right)\right.$ for $\mathrm{DE} \mathrm{P}_{1}$ and $\left(0.5 \AA, 0.0 \AA, 210^{\circ}, 3.5 \AA\right)$ for $\left.\mathrm{DE}_{-} \mathrm{P}_{2}\right)$ shows that the dispersion term, about 75 or $56 \%$ of the total binding energy, may play an important role in the DE-NMA parallel stacking (Table 1). To probe what determines the relative position of the two stacking components, we show in the 2-D $(x, y)$ PES, plotted with $\omega$ fixed at $120^{\circ}$ for $\mathrm{DE}_{-} \mathrm{P}_{1}$ (or at $210^{\circ}$ for $\mathrm{DE} \mathrm{P}_{2}$ ), that both the dispersion and the electrostatic terms have an energy minimum site near the total energy minimum site (Figure 7 and Figure S3 in the SI) but with the electrostatic

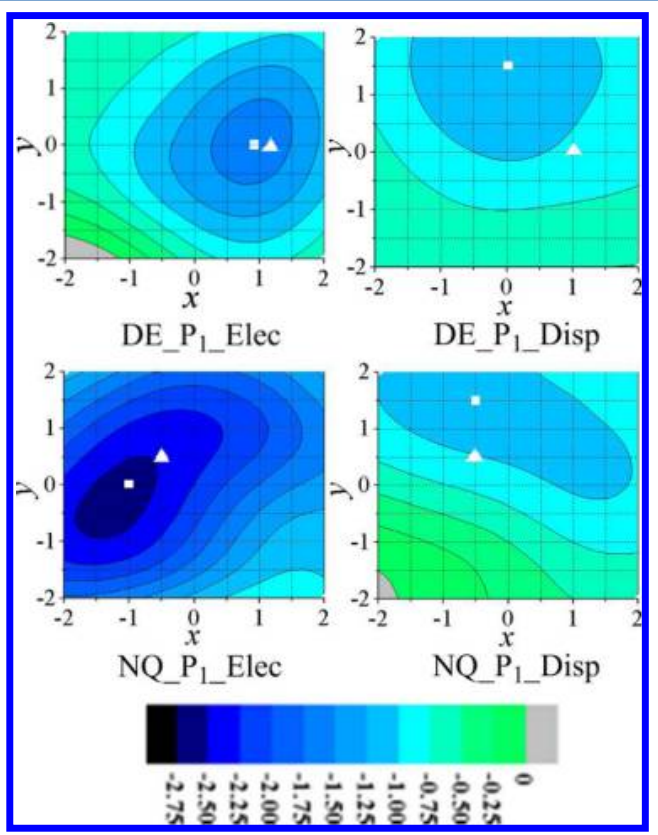

Figure 7. $(x, y)$ PESs of the electrostatic and dispersion components for the DE-NMA and NQ-NMA parallel stacking dimers with each contour corresponding to $0.25 \mathrm{kcal} / \mathrm{mol}$. ( $d=4.0 \AA$ and $\omega$ was fixed at its optimal value (Table 1).) The energy minimum site of the electrostatic PESs (left) or the dispersion PESs (right) was marked with a filled white square, while the total energy minimum site was marked with a filled white triangle

minimum site closer. Therefore, the optimal position is determined by both terms, but the electrostatic term appears to be more important. To see which term determines the relative orientation of the two stacking monomers, different energy components were plotted as a function of $\omega$ for DE $\mathrm{P}_{1}$ and DE_ $\mathrm{P}_{2}$ with $(x, y, d)$ fixed (Figure 6 and Figure S2 in the SI). The shape of the electrostatic curve is almost the same as the total energy curve with two minima at 120 and $240^{\circ}$ for $D_{1} P_{1}\left(60\right.$ and $210^{\circ}$ for $\left.\mathrm{DE}_{-} \mathrm{P}_{2}\right)$, suggesting that the orientation is determined primarily by the electrostatic 
interaction. It is found that the dipole of $\mathrm{DE}$, roughly along the direction of $\mathrm{C}_{1}-\mathrm{O}_{1}$, aligns not antiparallelly to that of NMA but tilts away by $\sim 40^{\circ}$ (Figure 4 ).

The electrostatic properties of asparagine/glutamine (NQ) are similar to the neutral DE. The ESPs are negative around $\mathrm{O}_{1}$ and $\mathrm{N}_{1}$ atoms but positive around other areas. The dipole of $\mathrm{NQ}$ is approximately along the direction of $\mathrm{C}_{1}-\mathrm{O}_{1}$, with a tilt angle of $7^{\circ}$ (Figure 5). Compared with $\mathrm{DE}$, the weaker electron-attractive ability of nitrogen in NQ would lead to less $\pi$ delocalization, indicating a more negative ESP on $\mathrm{O}_{1}$ and a larger molecular dipole along $\mathrm{C}_{1}-\mathrm{O}_{1}$ bond (4.5 $\mathrm{D}$ for $\mathrm{NQ}$ and $2.0 \mathrm{D}$ for $\mathrm{DE})$. The LMO-EDA analysis suggests that the electrostatic term and the dispersion term contribute the most to the total binding energy for the optimal NQ-NMA configuration $\left((x, y, \omega, d)\right.$ of $\left(-0.5 \AA, 0.5 \AA, 180^{\circ}, 3.6 \AA\right)$ for NQ $\mathrm{P}_{1}$ and $\left(1.5 \AA, 0.0 \AA, 150^{\circ}, 3.6 \AA\right.$ ) for NQ $\left.\mathrm{P}_{2}\right)$ (Table 1). Similar to DE-NMA complex, the 2-D $(x, y)$ PES, plotted with $\omega$ fixed at $180^{\circ}$ for NQ $\mathrm{P}_{1}$ (or at $150^{\circ}$ for NQ $\mathrm{P}_{2}$ ), shows that both the dispersion and the electrostatic terms have a minimum near the total energy minimum site but with the electrostatic term closer (Figures 7 and Figure S3 in the SI). By fixing the ( $x$, $y$ ) coordinates, the four LMO-EDA energy terms are plotted as a function of $\omega$ for NQ $P_{1}$ or $P_{2}$ models (Figure 6 and Figure $\mathrm{S} 2$ in the SI). It is found that the shape of the total energy curve is almost the same as the electrostatic curve, with the total energy change up to $4.1 \mathrm{kcal} / \mathrm{mol}$ for $\mathrm{NQ}_{1}$ or $3.9 \mathrm{kcal} / \mathrm{mol}$ for NQ $\mathrm{P}_{2}$ (Figure 6 and Figure S2 in the $\mathrm{SI}$ ). Different from the DE-NMA complex, the monomeric dipoles of NQ-NMA are aligned antiparallelly in both $\mathrm{NQ} \mathrm{P}_{1}$ and $\mathrm{NQ} \mathrm{P}_{2}$ optimal stacking. Thus, the optimal position of the parallel NQ-NMA is mainly determined by both electrostatic and dispersion, whereas the optimal orientation is determined by the electrostatic interaction.

For the $\mathrm{R}^{+}-\mathrm{NMA}$ parallel stacking model, the electrostatic term dominates the total interactions for both $\mathrm{P}_{1}$ and $\mathrm{P}_{2}$ optimal configurations $((x, y, \omega, d)$ of $(-0.5 \AA,-4.0 \AA$, $\left.240^{\circ}, 2.8 \AA\right)$ and $\left(-0.5 \AA,-4.0 \AA, 90^{\circ}, 3.5 \AA\right)$, respectively) (Table 1), where the arginine side chain with positive ESP prefers to locate above the most negative ESP area of NMA $\left(\mathrm{O}_{1}{ }^{\prime}\right)$ (Figure 4). The much lower $d$ value of $\mathrm{R}^{+} \mathrm{P}_{1}$ is attributed to the stacking configuration. As shown in Figures 3 and 4 , in the optimal configuration, the $\mathrm{R}^{+}$model moved $\sim 4 \AA$ away from the initial position, and only the $\mathrm{CH}_{3}$ group of $\mathrm{R}^{+}$is on top of NMA. Thus, the steric hindrance effect is small, mainly from the $\mathrm{CH}_{3}$. In addition, the methyl carbon $\mathrm{C}$ is not on the $\mathrm{N}_{1}-\mathrm{C}_{1}-\mathrm{N}_{2}$ plane of the $\mathrm{R}^{+}$monomer but points away from NMA with the dihedral $\angle \mathrm{N}_{1}-\mathrm{C}_{1}-\mathrm{N}_{2}-\mathrm{C}$ of $\sim 12^{\circ}$. In the $\mathrm{R}^{+} \mathrm{P}_{1}$ complex, the distance between the methyl carbon $\mathrm{C}$ of $\mathrm{R}^{+}$and NMA plane is $\sim 3.1 \AA$, which is $0.3 \AA$ larger than the interplanar distance, $d$. The dipole of $\mathrm{R}^{+}$model is perpendicular to $\mathrm{C}_{1}-\mathrm{N}_{1}$ bond, roughly along the $\mathrm{C}_{1}-\mathrm{N}_{3}$ bond vector (Figure 5 ). As shown in Figure 4, the direction of the $\mathrm{R}^{+}$dipole is approximately antiparallel to that of NMA in the optimal configurations. The total binding energy and the different energy components were plotted versus the $\omega$ angle $((x, y)$ fixed at $(-0.5 \AA,-4.0 \AA)$ ) (Figure 6 and Figure S2 in the SI). As the orientation of the $\mathrm{R}^{+}$dipole varies relative to that of NMA, the interaction energy can change by $0.6 \mathrm{kcal} / \mathrm{mol}$ for $\mathrm{R}^{+} \mathrm{P}_{1}$ or $1.0 \mathrm{kcal} / \mathrm{mol}$ for $\mathrm{R}^{+} \mathrm{P}_{2}$. The small variation indicates that the electrostatic energy is mainly from the charge-dipole interaction.

Optimal Configurations of the T-Shaped Stacking. The $(x, y)$ 2-D PES slice is shown for the $\mathrm{DE}^{-}-$, DE-, NQ-, and $\mathrm{R}^{+}-\mathrm{NMA}$ complexes (Figure $8, d=4.0 \AA$ and $\omega$ equals the optimal value (Table 1$)$ ). For the $\mathrm{DE}^{-}-\mathrm{NMA}$ complex, the

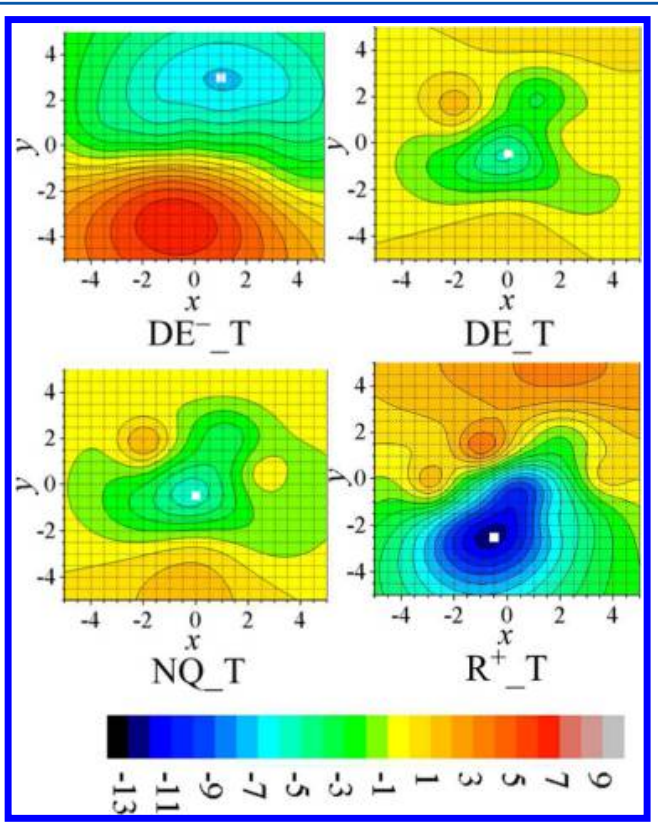

Figure 8. $(x, y)$ PES of the T-shaped dimers, each contour corresponding to $1 \mathrm{kcal} / \mathrm{mol}$. The distance $d$ was fixed at $4.0 \AA$ and the $\omega$ angle was fixed to its optimal value (Table 1). The minimum energy site was marked with a filled white square.

optimal configuration is that the $\mathrm{DE}^{-}$carboxyl group with negative ESPs locates above the most positive ESP area of NMA $\left(\mathrm{H}\right.$ of $\mathrm{N}_{1}{ }^{\prime}$ ), with the $\mathrm{N}_{1}{ }^{\prime}-\mathrm{H}$ bond aligning in between the two oxygen atoms $\mathrm{O}_{1}$ and $\mathrm{O}_{2}$ (Figure 9). For the DE-

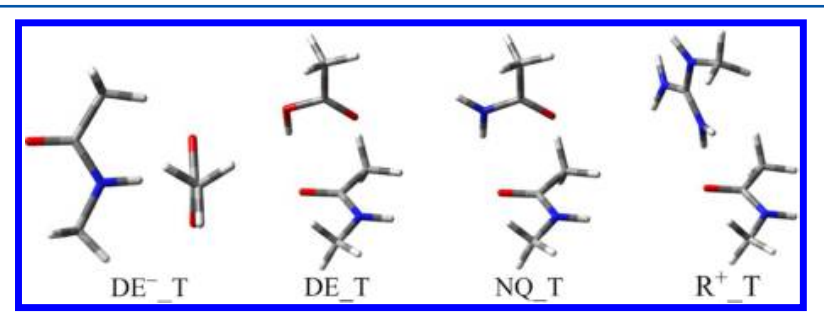

Figure 9. Optimal T-shaped stacking configurations obtained from the MP2/6-31+G(d,p) scan.

NMA optimal configuration $((x, y, \omega, d)$ of $(0.0 \AA,-0.5 \AA$, $\left.\left.180^{\circ}, 3.6 \AA\right)\right)$, the positive ESP area $\left(\mathrm{H}\right.$ of $\left.\mathrm{O}_{2}\right)$ of DE is located above the negative ESP area of NMA $\left(\mathrm{O}_{1}{ }^{\prime}\right)$ and a $\mathrm{O}_{2}-\mathrm{H} \cdots \mathrm{O}_{1}{ }^{\prime}$ hydrogen bond is formed, with the $\mathrm{O}_{2}-\mathrm{O}_{1}{ }^{\prime}$ distance of $3.2 \AA$ and the angle $\angle \mathrm{O}_{2}-\mathrm{H}-\mathrm{O}_{1}{ }^{\prime}$ of $173^{\circ}$. In addition, the dipole of DE aligns approximately antiparallell to that of NMA. As for the NQ-NMA complex, the optimal configuration is very similar to that of the DE-NMA dimer. A $\mathrm{N}_{1}-\mathrm{H} \cdots \mathrm{O}_{1}{ }^{\prime}$ hydrogen bond is formed, with the $\mathrm{N}_{1}-\mathrm{O}_{1}{ }^{\prime}$ distance of $3.2 \AA$ and the $\angle \mathrm{N}_{1}-\mathrm{H}-\mathrm{O}_{1}{ }^{\prime}$ angle of $162^{\circ}$, while the monomer dipoles are also aligned roughly antiparallelly. For the $\mathrm{R}^{+}-$ NMA complex with the optimal configuration at $(-0.5 \AA,-2.5$ $\AA$, $120^{\circ}, 3.4 \AA$ ), $\mathrm{R}^{+}$with the positive ESP sits near the negative ESP area of NMA $\left(\mathrm{O}_{1}{ }^{\prime}\right)$, whereas a hydrogen bond is formed between $\mathrm{N}_{1}$ and $\mathrm{O}_{1}^{\prime}$, with the $\mathrm{N}_{1}-\mathrm{O}_{1}^{\prime}$ distance of $2.8 \AA$ and $\angle \mathrm{N}_{1}-\mathrm{H}-\mathrm{O}_{1}{ }^{\prime}$ of $172^{\circ}$. The shorter distance between the heavy atoms of the hydrogen bond for $\mathrm{R}^{+} \mathrm{T}$ compared with that from DE_T is likely due to the smaller steric hindrance effect (Figure 
9). As reported in Table 1, the total interaction energies of the optimal configurations have the largest contributions from the electrostatic and polarization terms for the T-shaped stacking models. Therefore, all of the optimal T-shaped configurations are determined primarily by the electrostatic interaction. In addition, the $\mathrm{DE}^{-} \mathrm{T}$ complex has an optimal $d$ value $(2.2 \AA)$ much smaller than others (Table 1) due to the strong electrostatic attraction (Figure 9).

Binding Energy and the Solvation Effect. The CCSD(T)/CBS interaction energies for optimal configurations are reported (Table 2). For the parallel stacking, the absolute

Table 2. Solvation Effect on the Interaction Energies at $\operatorname{CCSD}(\mathrm{T}) / \mathrm{CBS}$ for Optimal Configurations ( $\mathrm{kcal} / \mathrm{mol}$ )

\begin{tabular}{lccc} 
& \multicolumn{3}{c}{$\Delta E_{\text {total }}$} \\
\cline { 2 - 4 } & $\varepsilon=1.0$ & $\varepsilon=4.0$ & $\varepsilon=80.0$ \\
DE_P & -4.6 & -3.2 & -2.7 \\
DE_P & -4.5 & -3.1 & -2.5 \\
NQ_P & -4.8 & -3.4 & -2.5 \\
NQ_P & -4.6 & -3.2 & -2.4 \\
DE $_{2}{ }_{2}$ & -7.8 & -3.1 & -1.9 \\
$\mathrm{R}^{+} \mathrm{P}_{1}$ & -11.0 & -3.9 & -1.7 \\
$\mathrm{R}^{+} \mathrm{P}_{2}$ & -8.6 & -3.1 & -1.4 \\
$\mathrm{DE}_{-} \mathrm{T}$ & -8.0 & -5.4 & -4.2 \\
$\mathrm{NQ}^{\mathrm{T}}$ & -8.0 & -4.8 & -3.3 \\
$\mathrm{DE}^{-} \mathrm{T}$ & -18.1 & -5.7 & -1.5 \\
$\mathrm{R}^{+}{ }^{-}$ & -18.0 & -8.7 & -5.5 \\
\hline
\end{tabular}

interaction energies are in the order of $\mathrm{R}^{+}>\mathrm{DE}^{-}>\mathrm{DE} \approx \mathrm{NQ}$. It has been reported that the interaction energy of benzene/ NMA parallel stacking is about $-2.5 \mathrm{kcal} / \mathrm{mol}$, ${ }^{30}$ smaller than that of the complexes reported in this work, where a large portion of the interaction energy comes from the electrostatics, for example, the charge-dipole and the dipole-dipole interactions (Table 1). As for the T-shaped stacking models, the interaction is in the order of $\mathrm{R}^{+} \mathrm{T}_{-} \approx \mathrm{DE}^{-} \mathrm{T}>\mathrm{DE} \_\mathrm{T} \approx$ NQ $T$, with the absolute interaction energies much larger than those of the parallel stacking models (Table 2).

Because the stacking occurs within a protein or on a protein surface, the PCM calculations with different dielectric constants (4.0 and 80.0) were carried out to mimic the solvation effect for the optimal configurations without further reoptimization (Table 2). It is worth noting that interaction energy at the $\mathrm{PCM} / \mathrm{CCSD}(\mathrm{T}) / \mathrm{CBS}$ was extrapolated in a similar way as in the gas-phase calculations for the purpose of direct comparison with the gas-phase results. The interaction energy in PCM is not very sensitive to the used basis set (aug-cc-pVDZ and aug-
cc-pVTZ), consistent with the gas-phase calculations (Figure $2)$. The extrapolated interaction energy at $\operatorname{PCM} / \operatorname{CCSD}(\mathrm{T}) /$ CBS is very similar to that at PCM/MP2/aug-cc-pVTZ. For the stacking at the low dielectric constant environment $(\varepsilon=4.0)$, all of the interactions are weakened. Taking the parallel stacking as an example, the binding energy increases (becomes less negative) by $4.7,7.1$ (5.5), 1.4 , and $1.4 \mathrm{kcal} / \mathrm{mol}$ for $\mathrm{DE}^{-}{ }_{-}$, $\mathrm{R}^{+} \mathrm{P}_{1}\left(\mathrm{P}_{2}\right), \mathrm{DE} \mathrm{P}$, and $\mathrm{NQ} \mathrm{P}$, respectively, meaning that the interactions of the charged models are weakened much more than those of the neutral ones. As a result, the interaction energies of the neutral complexes are nearly equivalent to those of the charged ones at $\varepsilon=4.0$, with the values ranging from approximately -3.1 to $-3.9 \mathrm{kcal} / \mathrm{mol}$ for the parallel stacking models. For the T-shaped configurations, the charged systems are also affected more than the neutral ones. The interaction energy of neutral DE_T $(-5.4 \mathrm{kcal} / \mathrm{mol})$ or NQ T $(-4.8$ $\mathrm{kcal} / \mathrm{mol})$ is nearly equal to that of $\mathrm{DE}^{-} \mathrm{T}(-5.7 \mathrm{kcal} / \mathrm{mol})$ but less negative than $\mathrm{R}^{+} \mathrm{T}(-8.7 \mathrm{kcal} / \mathrm{mol})$, which has a combination of the charge-dipole interaction and the intermolecular hydrogen bond. The energy difference between the parallel and the T-shaped stacking models also becomes smaller (Table 2). As the dielectric constant increases to 80.0, the interactions are further weakened for both the parallel stacking and the T-shaped stacking models. For the neutral complexes, the parallel stacking interaction energies change to about $-2.5 \mathrm{kcal} / \mathrm{mol}$, whereas the T-shaped stacking interaction energies change to -3 to $-4 \mathrm{kcal} / \mathrm{mol}$. The charged complexes generally have interactions weaker than the corresponding neutral ones, except for $\mathrm{R}^{+} \mathrm{T}$, which has the strongest interaction energy of $-5.5 \mathrm{kcal} / \mathrm{mol}$.

Survey of the Parallel Amide- and GuanidinoPeptide Plane Stacking in Proteins. The stacking of carboxyl-peptide plane has been surveyed in the pdb databank previously, and it shows that the parallel stacking is more common ( 0.28 interactions per protein structure) than the $\mathrm{T}$ shaped stacking. In this work, we performed a similar search for the parallel amide-peptide plane and guanidino-peptide plane stacking interactions. Three parameters are used to describe the stacking, namely, the distance, $d$, the angle, $\theta$, between the two planes (amide or guanidino group versus the peptide plane), and the distance, $r$, between the projection of $\mathrm{C}_{1}$ on the peptide plane and the origin $\mathrm{C}_{1}{ }^{\prime}$ (Figure 1). The cutoff values are $d<$ $5.0 \AA, \theta<20^{\circ}$, and $r<1.5 \AA$ for the parallel stacking. A total of 2577 and 3129 parallel stacking interactions are identified from a database of 6338 high-resolution X-ray structures, yielding an average of 0.41 and 0.49 interactions per structure for the amide - and guanidino-peptide plane stacking, respectively. An analysis of the distribution of the distance, $d$, shows a maximum

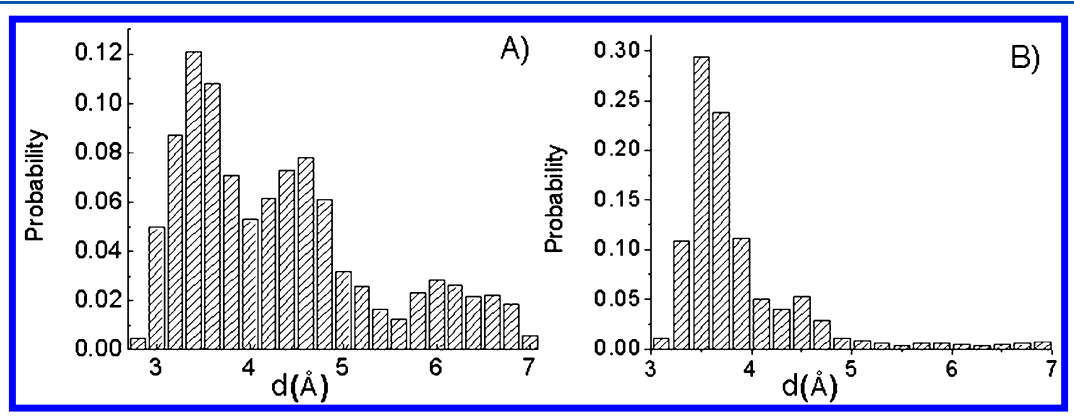

Figure 10. Histogram of the distance, $d$, (Figure 1) for the parallel amide-peptide plane (A) and guanidino-peptide plane stacking (B). The probability was corrected for the increase of volume at larger distance. 
occurrence at $d=3.4 \AA$ for the amide-peptide plane stacking and $d=3.5 \AA$ for the guanidino-peptide plane stacking (Figure $10)$, consistent with the QM calculations (Table 1).

\section{CONCLUSIONS}

Parallel and T-shaped stacking interactions of $\mathrm{DE}^{-}-$, DE-, $\mathrm{NQ}-$, and $\mathrm{R}^{+}-\mathrm{NMA}$ were investigated using the $\mathrm{QM}$ computational methods. For all stacking models, the electrostatic interaction plays an essential role in determining the optimal stacking position and the relative orientation. For the neutral complexes, the dispersion also contributes considerably to the stacking. In the gas phase, the stacking of the charged system is stronger than that of the neutral system, and the Tshaped stacking is generally stronger than the parallel stacking, but the solvation has a large effect in weakening the stacking, especially for the charged system. As the dielectric constant increases to 4.0, the parallel stacking energy is comparable for the charged and the neutral systems, whereas the T-shaped stacking energy is also similar for different systems (except $\mathrm{R}^{+}{ }_{-} \mathrm{T}$ ). As the dielectric constant increases further to 80.0, the interactions of the charged systems generally become weaker than the neutral ones (except $\mathrm{R}_{-}^{+} \mathrm{T}$ ).

\section{ASSOCIATED CONTENT}

\section{S Supporting Information}

MP2/6-31+G(d,p) $(x, y)$ PES for different parallel stacking models; different energy terms as a function of $\omega$; electrostatic and dispersion energies as a function of the $(x, y)$ coordinates; and the complete author list of ref 50 and51. This material is available free of charge via the Internet at http://pubs.acs.org.

\section{AUTHOR INFORMATION}

\section{Corresponding Author}

*E-mail: yaols@qibebt.ac.cn. Phone 86532 80662792. Fax: 86 53280662778.

\section{Notes}

The authors declare no competing financial interest.

\section{ACKNOWLEDGMENTS}

This work was partially supported by 100 Talent Project of Chinese Academy of Sciences, National Natural Science Foundation of China (grant nos. 21173247 and 31270785 to L.Y.) and the " 135 " Projects Fund of CAS-QIBEBT Director Innovation Foundation.

\section{REFERENCES}

(1) Salonen, L. M.; Ellermann, M.; Diederich, F. Aromatic Rings in Chemical and Biological Recognition: Energetics and Structures. Angew. Chem., Int. Ed. 2011, 50, 4808-4842.

(2) Schottel, B. L.; Chifotides, H. T.; Dunbar, K. R. Anion-pi Interactions. Chem. Soc. Rev. 2008, 37, 68-83.

(3) Hunter, C. A.; Lawson, K. R.; Perkins, J.; Urch, C. J. Aromatic Interactions. J. Chem. Soc., Perkin Trans. 2 2001, 5, 651-669.

(4) Riley, K. E.; Hobza, P. On the Importance and Origin of Aromatic Interactions in Chemistry and Biodisciplines. Acc. Chem. Res. 2013, 46, 927-936.

(5) Wheeler, S. E. Understanding Substituent Effects in Noncovalent Interactions Involving Aromatic Rings. Acc. Chem. Res. 2013, 46, 1029-1038.

(6) Daze, K. D.; Hof, F. The Cation-pi Interaction at Protein-Protein Interaction Interfaces: Developing and Learning from Synthetic Mimics of Proteins That Bind Methylated Lysines. Acc. Chem. Res. 2013, 46, 937-945.
(7) Macias, A. T.; Norton, J. E.; Evanseck, J. D. Impact of multiple cation-pi interactions upon calix[4]arene substrate binding and specificity. J. Am. Chem. Soc. 2003, 125, 2351-2360.

(8) Ma, J. C.; Dougherty, D. A. The Cation-pi Interaction. Chem. Rev. 1997, 97, 1303-1324.

(9) Dougherty, D. A. The Cation-pi Interaction. Acc. Chem. Res. 2013, 46, 885-893.

(10) Tsuzuki, S.; Honda, K.; Uchimaru, T.; Mikami, M.; Tanabe, K. The Magnitude of the $\mathrm{CH} /$ pi Interaction between Benzene and Some Model Hydrocarbons. J. Am. Chem. Soc. 2000, 122, 3746-3753.

(11) Tsuzuki, S.; Honda, K.; Uchimaru, T.; Mikami, M.; Tanabe, K. Origin of the Attraction and Directionality of the $\mathrm{NH} /$ pi Interaction: Comparison with $\mathrm{OH} / \mathrm{pi}$ and $\mathrm{CH} /$ pi Interactions. J. Am. Chem. Soc. 2000, 122, 11450-11458.

(12) Imai, Y. N.; Inoue, Y.; Nakanishi, I.; Kitaura, K. Amide-pi Interactions Between Formamide and Benzene. J. Comput. Chem. 2009, 30, 2267-2276.

(13) Riley, K. E.; Pitonak, M.; Jurecka, P.; Hobza, P. Stabilization and Structure Calculations for Noncovalent Interactions in Extended Molecular Systems Based on Wave Function and Density Functional Theories. Chem. Rev. 2010, 110, 5023-5063.

(14) Su, P. F.; Jiang, Z.; Chen, Z. C.; Wu, W. Energy Decomposition Scheme Based on the Generalized Kohn-Sham Scheme. J. Phys. Chem. A 2014, 118, 2531-2542.

(15) Su, P. F.; Li, H. Energy Decomposition Analysis of Covalent Bonds and Intermolecular Interactions. J. Chem. Phys. 2009, 131, 014102.

(16) Jeziorski, B.; Moszynski, R.; Szalewicz, K. Perturbation-Theory Approach to Intermolecular Potential-Energy Surfaces of Van-DerWaals Complexes. Chem. Rev. 1994, 94, 1887-1930.

(17) Sinnokrot, M. O.; Sherrill, C. D. High-Accuracy Quantum Mechanical Studies of pi-pi Interactions in Benzene Dimers. J. Phys. Chem. A 2006, 110, 10656-10668.

(18) Sinnokrot, M. O.; Sherrill, C. D. Highly Accurate Coupled Cluster Potential Energy Curves for the Benzene Dimer: Sandwich, TShaped, and Parallel-Displaced Configurations. J. Phys. Chem. A 2004, 108, 10200-10207.

(19) Pitonak, M.; Neogrady, P.; Rezac, J.; Jurecka, P.; Urban, M.; Hobza, P. Benzene Dimer: High-Level Wave Function and Density Functional Theory Calculations. J. Chem. Theory Comput. 2008, 4, $1829-1834$.

(20) Podeszwa, R.; Bukowski, R.; Szalewicz, K. Potential Energy Surface for the Benzene Dimer and Perturbational Analysis of pi-pi Interactions. J. Phys. Chem. A 2006, 110, 10345-10354.

(21) Krishtal, A.; Senet, P.; Van Alsenoy, C. Local Softness, Softness Dipole, and Polarizabilities of Functional Groups: Application to the Side Chains of the 20 Amino Acids. J. Chem. Phys. 2009, 131, 044312.

(22) Hohenstein, E. G.; Sherrill, C. D. Wavefunction Methods for Noncovalent Interactions. Wiley Interdiscip. Rev.: Comput. Mol. Sci. 2012, 2, 304-326.

(23) Sherrill, C. D. Energy Component Analysis of pi Interactions. Acc. Chem. Res. 2013, 46, 1020-1028.

(24) Riley, K. E.; Merz, K. M. Role of Solvation in the Energy Stabilization inside the Hydrophobic Core of the Protein Rubredoxin. J. Phys. Chem. B 2006, 110, 15650-15653.

(25) Vondrasek, J.; Bendova, L.; Klusak, V.; Hobza, P. Unexpectedly Strong Energy Stabilization inside the Hydrophobic Core of Small Protein Rubredoxin Mediated by Aromatic Residues: Correlated Ab Initio Quantum Chemical Calculations. J. Am. Chem. Soc. 2005, 127, 2615-2619.

(26) Bendova-Biedermannova, L.; Hobza, P.; Vondrasek, J. Identifying Stabilizing Key Residues in Proteins Using Interresidue Interaction Energy Matrix. Proteins: Struct., Funct., Bioinf. 2008, 72, 402-413.

(27) Berka, K.; Laskowski, R. A.; Hobza, P.; Vondrasek, J. Energy Matrix of Structurally Important Side-Chain/Side-Chain Interactions in Proteins. J. Chem. Theory Comput. 2010, 6, 2191-2203. 
(28) Cerny, J.; Vondrasek, J.; Hobza, P. Loss of Dispersion Energy Changes the Stability and Folding/Unfolding Equilibrium of the TrpCage Protein. J. Phys. Chem. B 2009, 113, 5657-5660.

(29) Vondrasek, J.; Kubar, T.; Jenney, F. E.; Adams, M. W. W.; Kozisek, M.; Cerny, J.; Sklenar, V.; Hobza, P. Dispersion Interactions Govern the Strong Thermal Stability of a Protein. Chem.-Eur. J. 2007, 13, 9022-9027.

(30) Bendova, L.; Jurecka, P.; Hobza, P.; Vondrasek, J. Model of Peptide Bond-Aromatic Ring Interaction: Correlated $\mathrm{Ab}$ Initio Quantum Chemical Study. J. Phys. Chem. B 2007, 111, 9975-9979.

(31) Neidigh, J. W.; Fesinmeyer, R. M.; Andersen, N. H. Designing a 20-Residue Protein. Nat. Struct. Biol. 2002, 9, 425-430.

(32) Harder, M.; Kuhn, B.; Diederich, F. Efficient Stacking on Protein Amide Fragments. ChemMedChem. 2013, 8, 397-404.

(33) James, W. H.; Muller, C. W.; Buchanan, E. G.; Nix, M. G. D.; Guo, L.; Roskop, L.; Gordon, M. S.; Slipchenko, L. V.; Gellman, S. H.; Zwier, T. S. Intramolecular Amide Stacking and Its Competition with Hydrogen Bonding in a Small Foldamer. J. Am. Chem. Soc. 2009, 131, 14243-14245.

(34) James, W. H.; Buchanan, E. G.; Guo, L.; Geman, S. H.; Zwier, T. S. Competition between Amide Stacking and Intramolecular H Bonds in gamma-Peptide Derivatives: Controlling Nearest-Neighbor Preferences. J. Phys. Chem. A 2011, 115, 11960-11970.

(35) Sherrill, C. D.; Takatani, T.; Hohenstein, E. G. An Assessment of Theoretical Methods for Nonbonded Interactions: Comparison to Complete Basis Set Limit Coupled-Cluster Potential Energy Curves for the Benzene Dimer, the Methane Dimer, Benzene-Methane, and Benzene-H2S. J. Phys. Chem. A 2009, 113, 10146-10159.

(36) He, C.; Chen, J.; An, L.; Wang, Y.; Shu, Z.; Yao, L. CarboxylPeptide Plane Stacking Is Important for the Stabilization of the Buried E305 of Trichoderma Reesei Cel5A. J. Chem. Inf. Model. 2015, 55, 104113.

(37) Bush, J.; Makhatadze, G. I. Statistical Analysis of Protein Structures Suggests that Buried Ionizable Residues in Proteins are Hydrogen Bonded or Form Salt Bridges. Proteins: Struct., Funct., Bioinf. 2011, 79, 2027-2032.

(38) Kajander, T.; Kahn, P. C.; Passila, S. H.; Cohen, D. C.; Lehtio, L.; Adolfsen, W.; Warwicker, J.; Schell, U.; Goldman, A. Buried Charged Surface in Proteins. Structure 2000, 8, 1203-1214.

(39) Warshel, A.; Sharma, P. K.; Kato, M.; Parson, W. W. Modeling Electrostatic Effects in Proteins. Biochim. Biophys. Acta, Proteins Proteomics 2006, 1764, 1647-1676.

(40) Tissot, A. C.; Vuilleumier, S.; Fersht, A. R. Importance of Two Buried Salt Bridges in the Stability and Folding Pathway of Barnase. Biochemistry 1996, 35, 6786-6794.

(41) Trevino, S. R.; Gokulan, K.; Newsom, S.; Thurlkill, R. L.; Shaw, K. L.; Mitkevich, V. A.; Makarov, A. A.; Sacchettini, J. C.; Scholtz, J. M.; Pace, C. N. Asp79 Makes a Large, Unfavorable Contribution to the Stability of RNase Sa. J. Mol. Biol. 2005, 354, 967-978.

(42) Jayasimha, P.; Shanmuganathan, A.; Suladze, S.; Makhatadze, G. I. Contribution of Buried Aspartic Acid to the Stability of the PDZ2 Protein. J. Chem. Thermodyn. 2012, 52, 64-68.

(43) Headgordon, M.; Pople, J. A.; Frisch, M. J. Mp2 Energy Evaluation by Direct Methods. Chem. Phys. Lett. 1988, 153, 503-506.

(44) Petersson, G. A.; Bennett, A.; Tensfeldt, T. G.; Allaham, M. A.; Shirley, W. A.; Mantzaris, J. A Complete Basis Set Model Chemistry 0.1. The Total Energies of Closed-Shell Atoms and Hydrides of the 1st-Row Elements. J. Chem. Phys. 1988, 89, 2193-2218.

(45) Simon, S.; Duran, M.; Dannenberg, J. J. How Does Basis Set Superposition Error Change the Potential Surfaces for Hydrogen Bonded Dimers? J. Chem. Phys. 1996, 105, 11024-11031.

(46) Kendall, R. A.; Dunning, T. H.; Harrison, R. J. ElectronAffinities of the 1st-Row Atoms Revisited - Systematic Basis-Sets and Wave-Functions. J. Chem. Phys. 1992, 96, 6796-6806.

(47) Woon, D. E.; Dunning, T. H. Gaussian-Basis Sets for Use in Correlated Molecular Calculations 0.3. The Atoms Aluminum through Argon. J. Chem. Phys. 1993, 98, 1358-1371.

(48) Halkier, A.; Helgaker, T.; Jorgensen, P.; Klopper, W.; Koch, H.; Olsen, J.; Wilson, A. K. Basis-Set Convergence in Correlated
Calculations on Ne, N-2, and H2O. Chem. Phys. Lett. 1998, 286, 243-252.

(49) Tomasi, J.; Mennucci, B.; Cammi, R. Quantum Mechanical Continuum Solvation Models. Chem. Rev. 2005, 105, 2999-3093.

(50) Frisch, M. J.; Trucks, G. W.; Schlegel, H. B.; Scuseria, G. E.; Robb, M. A.; Cheeseman, J. R.; Scalmani, G.; Barone, V.; Mennucci, B.; Petersson, G. A. et al. Gaussian 09, revision B.01; Gaussian, Inc.: Wallingford, CT, 2010.

(51) Schmidt, M. W.; Baldridge, K. K.; Boatz, J. A.; Elbert, S. T.; Gordon, M. S.; Jensen, J. H.; Koseki, S.; Matsunaga, N.; Nguyen, K. A.; $\mathrm{Su}, \mathrm{S}$. J.; et al. General Atomic and Molecular Electronic-Structure System. J. Comput. Chem. 1993, 14, 1347-1363.

(52) Gordon, M. S.; Schmidt, M. W. Advances in Electronic Structure Theory: GAMESS a Decade Later; Elsevier; Amsterdam, The Netherlands, 2005.

(53) Aikens, C. M.; Webb, S. P.; Bell, R. L.; Fletcher, G. D.; Schmidt, M. W.; Gordon, M. S. A Derivation of the Frozen-Orbital Unrestricted Open-Shell and Restricted Closed-Shell Second-Order Perturbation Theory Analytic Gradient Expressions. Theor. Chem. Acc. 2003, 110, 233-253.

(54) Wang, G. L.; Dunbrack, R. L. PISCES: a Protein Sequence Culling Server. Bioinformatics 2003, 19, 1589-1591.

(55) Humphrey, W.; Dalke, A.; Schulten, K. VMD: Visual Molecular Dynamics. J. Mol. Graph. Model. 1996, 14, 33-38. 